\title{
Asymptotic scaling from strong coupling
}

\author{
Massimo Campostrini, Paolo Rossi, and Ettore Vicari \\ Dipartimento di Fisica dell'Università and I.N.F.N., I-56126 Pisa, Italy
}

\begin{abstract}
Strong-coupling analysis of two-dimensional chiral models, extended to 15th order, allows for the identification of a scaling region where known continuum results are reproduced with great accuracy, and asymptotic scaling predictions are fulfilled. The properties of the large- $N$ second-order phase transition are quantitatively investigated.
\end{abstract}


Recent developments in the analytical and numerical study of lattice two-dimensional $\mathrm{SU}(N) \times \mathrm{SU}(N)$ principal chiral models have shown the existence of a scaling region, where continuum predictions for dimensionless ratios of physical quantities are substantially verified [1].

The scaling region begins at very small values of the correlation length: $\xi \gtrsim 2$, well within the region of convergence of the strong-coupling series. Moreover by performing a nonperturbative change of variables [2] from the standard Lagrangian (inverse) coupling $\beta$ to

$$
\beta_{E}=\frac{N^{2}-1}{8 N^{2} E},
$$

where $E$ is the internal energy of the system (which can be measured in a Monte Carlo simulation), one can find agreement in the whole scaling region between the measured mass scale and the prediction for the mass gap $-\Lambda$ parameter ratio offered by the two-loop continuum renormalization group supplemented by a Bethe-Ansatz evaluation [3] ]. As a matter of fact, this may be thought as evidence for asymptotic scaling within the strongcoupling regime.

We therefore felt motivated for a significant effort in improving our analytical knowledge of the strong-coupling series for principal chiral models, in order to check the accuracy of predictions for physical (continuum) quantities solely based on strong-coupling computations.

As a byproduct, strong-coupling series can be analyzed to investigate the critical behavior of the $N=\infty$ theory, where Monte Carlo data seem to indicate the existence of a transition at finite $\beta$.

Strong-coupling calculations for matrix models are best performed by means of the character expansion. Even the character expansion however eventually runs into almost intractable technical difficulties, related to two concurrent phenomena:

1. the proliferation of configurations contributing to large orders of the series, whose number essentially grows like that of non-backtracking random walks;

2. the appearance of topologically nontrivial configurations corresponding to group integrations that cannot be performed by applying the orthogonality of characters and the decomposition of their products into sums.

We shall devote an extended paper to a complete presentation of our 15th-order strongcoupling character expansions for arbitrary $\mathrm{U}(N)$ groups (extension to $\mathrm{SU}(N)$ is then obtained by the techniques discussed in Refs. [1] and [1]). We only mention that we had to rely on a mixed approach: problem 1 above was tackled by a computer program generating all non-backtracking random walks, computing their correct multiplicities, and classifying them according to their topology; problem 2, requiring the analytical evaluation of many classes of nontrivial group integrals, and extraction of their connected contribution to the relevant physical quantities, was solved by more conventional algebraic techniques.

We pushed our computational techniques close to their limit; in order to extend our results to higher orders, a more algorithmic approach would be in order, especially for recognition of diagram topologies and group integration. 
In the present letter we shall only exhibit the results we obtained by taking the large- $N$ limit of all the quantities we computed. In the strong-coupling domain, convergence to $N=$ $\infty$ is usually fast, and therefore large- $N$ results are a good illustration of a phenomenology that repeats itself (with small corrections) for finite values of $N$.

Starting from the standard nearest-neighbour interaction

$$
S_{L}=-2 N \beta \sum_{x, \mu} \operatorname{Re} \operatorname{Tr}\left\{U(x) U^{\dagger}(x+\mu)\right\},
$$

we computed the large- $N$ free energy

$$
F=\frac{1}{N^{2}} \ln \int d U_{n} \exp \left(-S_{L}\right)
$$

to 18 th order in the expansion in powers of $\beta$. The resulting series is

$$
\begin{aligned}
F=\beta^{2} & +2 \beta^{4}+4 \beta^{6}+19 \beta^{8}+96 \beta^{10}+604 \beta^{12}+4036 \beta^{14} \\
& +\frac{58471}{2} \beta^{16}+\frac{663568}{3} \beta^{18}+\mathrm{O}\left(\beta^{20}\right) .
\end{aligned}
$$

The two-point Green's functions, normalized to obtain a finite large- $N$ limit, are

$$
G(x)=\frac{1}{N} \operatorname{Tr}\left\{U^{\dagger}(x) U(0)\right\} .
$$

We computed all nontrivial two-point Green's functions up to $\mathrm{O}\left(\beta^{15}\right)$. The corresponding information is appropriately summarized by introducing the lattice Fourier transform

$$
\tilde{G}(p)=\sum_{x} G(x) \exp (i p \cdot x)
$$

and evaluating the inverse propagator

$$
\begin{aligned}
\tilde{G}^{-1}(p)=A_{0} & +\hat{p}^{2} A_{1}+\left(\left(\hat{p}^{2}\right)^{2}-\hat{p}^{4}\right) A_{2,0}+\hat{p}^{4} A_{2,2}+\hat{p}^{2}\left(\left(\hat{p}^{2}\right)^{2}-\hat{p}^{4}\right) A_{3,1}+\hat{p}^{6} A_{3,3} \\
& +\left(\left(\hat{p}^{2}\right)^{2}-\hat{p}^{4}\right)^{2} A_{4,0}+\hat{p}^{4}\left(\left(\hat{p}^{2}\right)^{2}-\hat{p}^{4}\right) A_{4,2}+\hat{p}^{8} A_{4,4}+\hat{p}^{2}\left(\left(\hat{p}^{2}\right)^{2}-\hat{p}^{4}\right)^{2} A_{5,1} \\
& +\hat{p}^{6}\left(\left(\hat{p}^{2}\right)^{2}-\hat{p}^{4}\right) A_{5,3}+\ldots,
\end{aligned}
$$

where

$$
\begin{aligned}
A_{0}=1- & 4 \beta+4 \beta^{2}-4 \beta^{3}+12 \beta^{4}-28 \beta^{5}+52 \beta^{6}-132 \beta^{7}+324 \beta^{8}-908 \beta^{9}+2020 \beta^{10} \\
& -6284 \beta^{11}+15284 \beta^{12}-48940 \beta^{13}+116596 \beta^{14}-393052 \beta^{15}+\mathrm{O}\left(\beta^{16}\right), \\
A_{1}= & \beta\left(1+\beta^{2}+7 \beta^{4}+4 \beta^{5}+33 \beta^{6}+32 \beta^{7}+243 \beta^{8}+324 \beta^{9}+1819 \beta^{10}+2520 \beta^{11}\right. \\
& \left.+14859 \beta^{12}+23124 \beta^{13}+123767 \beta^{14}\right)+\mathrm{O}\left(\beta^{16}\right) \\
A_{2,0}=- & \beta^{6}\left(1+6 \beta^{2}+8 \beta^{3}+57 \beta^{4}+116 \beta^{5}+500 \beta^{6}+1152 \beta^{7}+5173 \beta^{8}+11600 \beta^{9}\right) \\
& +\mathrm{O}\left(\beta^{16}\right) \\
A_{2,2}=- & -2 \beta^{8}\left(1+2 \beta+12 \beta^{2}+35 \beta^{3}+121 \beta^{4}+408 \beta^{5}+1424 \beta^{6}+4244 \beta^{7}\right)+\mathrm{O}\left(\beta^{16}\right), \\
A_{3,1}= & \beta^{9}\left(1+\frac{29}{2} \beta^{2}+26 \beta^{3}+144 \beta^{4}+488 \beta^{5}+1802 \beta^{6}\right)+\mathrm{O}\left(\beta^{16}\right),
\end{aligned}
$$




$$
\begin{aligned}
& A_{3,3}=2 \beta^{11}\left(1+2 \beta+20 \beta^{2}+72 \beta^{3}+272 \beta^{4}\right)+\mathrm{O}\left(\beta^{16}\right) \\
& A_{4,0}=-\beta^{12}\left(\frac{5}{2}+37 \beta^{2}+84 \beta^{3}\right)+\mathrm{O}\left(\beta^{16}\right) \\
& A_{4,2}=-\beta^{12}\left(1+32 \beta^{2}+64 \beta^{3}\right)+\mathrm{O}\left(\beta^{16}\right) \\
& A_{4,4}=-2 \beta^{14}(1+2 \beta)+\mathrm{O}\left(\beta^{16}\right) \\
& A_{5,1}=7 \beta^{15}+\mathrm{O}\left(\beta^{16}\right) \\
& A_{5,3}=\beta^{15}+\mathrm{O}\left(\beta^{16}\right)
\end{aligned}
$$

A number of physically interesting quantities can be extracted from $\tilde{G}^{-1}(p)$. In particular the magnetic susceptibility is

$$
\begin{aligned}
\chi=\sum_{x} G(x)=\frac{1}{A_{0}}=1 & +4 \beta+12 \beta^{2}+36 \beta^{3}+100 \beta^{4}+284 \beta^{5}+796 \beta^{6}+2276 \beta^{7}+6444 \beta^{8} \\
& +18572 \beta^{9}+53292 \beta^{10}+155500 \beta^{11}+451516 \beta^{12}+1330796 \beta^{13} \\
& +3904924 \beta^{14}+11617404 \beta^{15}+\mathrm{O}\left(\beta^{16}\right)
\end{aligned}
$$

while the second-moment definition of the correlation length leads to

$$
\begin{aligned}
M_{G}^{2}=\frac{1}{\left\langle x^{2}\right\rangle_{G}}=\frac{A_{0}}{A_{1}}=\frac{1}{\beta} & -4+3 \beta+2 \beta^{3}-4 \beta^{4}+12 \beta^{5}-40 \beta^{6}+84 \beta^{7}-296 \beta^{8}+550 \beta^{9} \\
& -1904 \beta^{10}+3316 \beta^{11}-15288 \beta^{12}+28016 \beta^{13}+\mathrm{O}\left(\beta^{14}\right)
\end{aligned}
$$

and the corresponding wavefunction renormalization is $Z_{G}=A_{1}^{-1}$.

Moreover, by solving the equation

$$
\tilde{G}^{-1}\left(p_{x}=i \mu_{\mathrm{s}}, p_{y}=0\right)=0,
$$

we can compute the wall-wall correlation length, i.e. the true mass-gap $\mu_{\mathrm{s}}$; Eq. (11) is algebraic in

$$
\begin{aligned}
M_{\mathrm{s}}^{2}=2\left(\cosh \mu_{\mathrm{s}}-1\right)=\frac{1}{\beta} & -4+3 \beta+2 \beta^{3}-4 \beta^{4}+10 \beta^{5}-28 \beta^{6}+48 \beta^{7}-206 \beta^{8} \\
& +352 \beta^{9}-1510 \beta^{10}+2354 \beta^{11}+\mathrm{O}\left(\beta^{12}\right) .
\end{aligned}
$$

By solving

$$
\tilde{G}^{-1}\left(p_{x}=i \mu_{\mathrm{d}} / \sqrt{2}, p_{y}=i \mu_{\mathrm{d}} / \sqrt{2}\right)=0
$$

we find the diagonal wall-wall correlation length

$$
M_{\mathrm{d}}^{2}=4\left(\cosh \frac{\mu_{\mathrm{d}}}{\sqrt{2}}-1\right)=\frac{1}{\beta}-4+3 \beta+\frac{3}{2} \beta^{3}-3 \beta^{5}-\frac{169}{8} \beta^{7}-\frac{881}{4} \beta^{9}+\mathrm{O}\left(\beta^{11}\right) .
$$

In order to obtain the highest-order contribution to $M_{\mathrm{s}}^{2}$, it was necessary to compute a few (long-distance) Green's functions to 16th and 17th order.

Lattice chiral models have a peak in the specific heat 


$$
C=\frac{1}{N} \frac{d E}{d T}, \quad T=\frac{1}{N \beta}
$$

which becomes more and more pronounced with increasing $N$ [1]. Fig. 1] shows Monte Carlo data for the specific heat of $\mathrm{SU}(N)$ models for $N=21,30$, and $\mathrm{U}(N)$ models for $N=15,21$. (We recall that $\mathrm{U}(N)$ models at finite $N$ should experience a phase transition with a pattern similar to the XY model, but its location is beyond the specific heat peak). With increasing $N$, the positions of the peaks in $\mathrm{SU}(N)$ and $\mathrm{U}(N)$ seems to approach the same value of $\beta$, consistently with the fact that $\mathrm{SU}(N)$ and $\mathrm{U}(N)$ models must have the same large- $N$ limit. This should be considered an indication of a phase transition at $N=\infty$; a rough extrapolation of the $C$ data indicates a critical coupling $\beta_{c} \simeq 0.306$. Extrapolating the values of $\xi_{G}=1 / M_{G}$ and $\beta_{E}$ at the peak of $C$ to $N=\infty$, we obtain respectively $\xi_{G}^{(c)} \simeq 2.8$ and $\beta_{E}^{(c)} \simeq 0.220$.

The above picture is confirmed by an analysis of the large- $N$ 18th order strong-coupling series of $C$, based on the method of the integral approximants [5.6]. We indeed obtained quite stable results showing the critical behavior

$$
C \sim\left|\beta-\beta_{c}\right|^{-\alpha}
$$

with $\beta_{c} \cong 0.3054$ and $\alpha \cong 0.21$, in agreement with the extrapolation of Monte Carlo data. Fig. 1 shows that simulation data of $C$ approach, for growing $N$, the strong-coupling determination.

In spite of the existence of a phase transition at $N=\infty$, Monte Carlo data show scaling and asymptotic scaling (in the $\beta_{E}$ scheme) even for $\beta$ smaller then the peak of the specific heat, suggesting an effective decoupling of the modes responsible for the phase transition from those determining the physical continuum limit; this phenomenon motivated us to use the strong-coupling approach to test scaling and asymptotic scaling. In Fig. 2 we plot the dimensionless ratio $\mu_{s} / M_{G}$ vs. the correlation length $\xi_{G} \equiv 1 / M_{G}$, as obtained from our strong-coupling series. Notice the stability of the curve for a large region of values of $\xi_{G}$ and the good agreement (within a few per mille) with the continuum large- $N$ value extrapolated by Monte Carlo data $\mu_{s} / M_{G}=0.991(1)$ [1].

In order to test asymptotic scaling we perform the variable change indicated in Eq.(四), evaluating the energy from its strong-coupling series

$$
E=1-\frac{1}{4} \frac{\partial F}{\partial \beta}=1-G((1,0))
$$

(cf. Eq. (3)). The asymptotic scaling formula for the mass gap in the $\beta_{E}$ scheme is, in the large- $N$ limit,

$$
\mu \cong \sqrt{\frac{\pi}{e}} 16 \exp \left(\frac{\pi}{4}\right) \Lambda_{E, 2 l}\left(\beta_{E}\right), \quad \Lambda_{E, 2 l}\left(\beta_{E}\right)=\sqrt{8 \pi \beta_{E}} \exp \left(-8 \pi \beta_{E}\right)
$$

$\beta_{E}$ can be expressed as a strong-coupling expanded function of $\beta$ by means of Eq. (17). In Fig. 3 the strong-coupling estimates of $\mu_{s} / \Lambda_{E, 2 l}$ and $M_{G} / \Lambda_{E, 2 l}$ are plotted vs. $\beta_{E}$, for a region of coupling corresponding to correlation lengths $1 \lesssim \xi_{G} \lesssim 3$. These quantities agree with the exact continuum prediction within $5 \%$ in the whole region. 
Since the large- $N \beta$-function in the $\beta_{E}$ scheme is not singular (as shown by Monte Carlo data [1] and strong-coupling analysis) and the specific heat has a divergence at $\beta_{c}$, then the relationship

$$
\beta_{E}\left(T_{E}\right)=\frac{8 N^{2}}{N^{2}-1} C(T) \beta_{L}(T)
$$

between the $\beta$-function in the standard scheme $\beta_{L}(T)$ and in the $\beta_{E}$ scheme $\beta_{E}\left(T_{E}\right)$ leads to an non-analytical zero of $\beta_{L}(T)$ at $\beta_{c}: \beta_{L}(T) \sim\left|\beta-\beta_{c}\right|^{\alpha}$, explaining the observed behavior in $\beta$ of the Monte Carlo data for the mass gap at large $N$ [1].

This phenomenon is further confirmed by an analysis of the strong-coupling series of $\chi$ and $M_{G}^{2}$. Assuming they are well-behaved functions of the energy, we should have

$$
\frac{d \ln \chi}{d \beta} \sim \frac{d \ln M_{G}^{2}}{d \beta} \sim\left|\beta-\beta_{c}\right|^{-\alpha}
$$

Analyzing the corresponding series by a modified integral approximant scheme which forces the approximant to have a singularity at $\beta \simeq 0.3054$, we found a critical behavior consistent with Eq. (20) $(\alpha \simeq 0.2)$. 


\section{REFERENCES}

[1] P. Rossi and E. Vicari, "Two dimensional $\mathrm{SU}(N) \times \mathrm{SU}(\mathrm{N})$ Chiral Models on the Lattice (II): the Green's Function", Pisa preprint IFUP-TH 8/94, Phys. Rev. D, in press.

[2] G. Parisi, in High Energy Physics 1980, Proceedings of the XXth Conference on High Energy Physics, Madison, Wisconsin, 1980, edited by L. Durand and L. G. Pondrom, AIP Conf. Proc. No. 68 (AIP, New York, 1981).

[3] J. Balog, S. Naik, F. Niedermayer, and P. Weisz, Phys. Rev. Lett. 69, 873 (1992).

[4] F. Green and S. Samuel, Nucl. Phys. B190, 113 (1981).

[5] D. L. Hunter and G. A. Baker Jr., Phys. Rev. B 49 (1979) 3808.

[6] M. E. Fisher and H. Au-Yang, J. Phys. A12 (1979) 1677. 


\section{FIGURES}

FIG. 1. Specific heat vs. $\beta$. The solid line represents the resummation of the strong-coupling series, whose estimate of the critical $\beta$ is indicated by the vertical dashed lines.

FIG. 2. $\mu_{s} / M_{G}$ vs. $\xi_{G} \equiv 1 / M_{G}$. The dashed line represents the continuum result from Monte Carlo data.

FIG. 3. Asymptotic scaling test by using strong-coupling estimates. The dotted line represents the exact result (18). 
This figure "fig1-1.png" is available in "png" format from: http://arxiv.org/ps/hep-lat/9406014v1 
This figure "fig1-2.png" is available in "png" format from: http://arxiv.org/ps/hep-lat/9406014v1 